Editorial

\title{
Mitochondrial oxidative phosphorylation is linked to T-cell exhaustion
}

Shuai Jiang

Mounting evidence indicates that metabolic reprogramming is not only a hallmark of tumor cells, but also critical for various immune cells, including $\mathrm{T}$ cells [1]. Studies of immunometabolism demonstrate that the functional roles of $\mathrm{T}$-cell subsets are linked to different metabolic pathways and key factors (e.g., metabolic enzymes and metabolites), which can modulate T-cell development, differentiation, and activation [2]. In certain pathological settings, such as tumors, T-cell populations called "exhausted T cells" are unable to exert their functional roles. This phenomenon is similar to observations in chronic viral infection; however, the underlining molecular mechanism and its corresponding metabolic alterations remains unclear. It is presently unknown how repetitive antigen challenge leads to T-cell exhaustion, as exhibited by self-renewal ability failure. Thus, there is substantial interest in elucidating how metabolism represents a key link to T-cell exhaustion.

In the new research article "Impaired mitochondrial oxidative phosphorylation limits the self-renewal of $\mathrm{T}$ cells exposed to persistent antigen" [3], Dr. Thompson's group at Memorial Sloan Kettering Cancer Center cha- racterized the role of metabolic reprogramming in T-cell exhaustion using mouse models of melanoma (Figure 1). They found that persistent antigen challenge led to a shift in metabolic reprogramming, which was critical for restricting T-cell self-renewal and proliferation. The authors observed that exhausted T cells exhibited higher glycolysis, impaired mitochondrial functions, and lower nucleotide biosynthesis. Further characterizing the molecular mechanism of mitochondrial dysfunction in exhausted $\mathrm{T}$ cells, they detected significantly enhanced activity of the key regulator of T-cell exhaustion NFAT, and demonstrated that antigen-induced ROS activity was tightly linked to T-cell exhaustion. Moreover, they proved that antioxidant treatment restored metabolic $\mathrm{T}$ cell function and self-renewal in exhausted $\mathrm{T}$ cells during chronic antigen stimulation, both in vitro and in vivo. This study highlights that oxidative force is an aftereffect of chronic antigen-driven mitochondrial dysfunction, which can diminish T-cell proliferation and self-renewal capacities in vitro and in vivo, suggesting that treatment with a functional sustaining redox balance may restore $\mathrm{T}$ cells from "exhausted" to "effective" status.

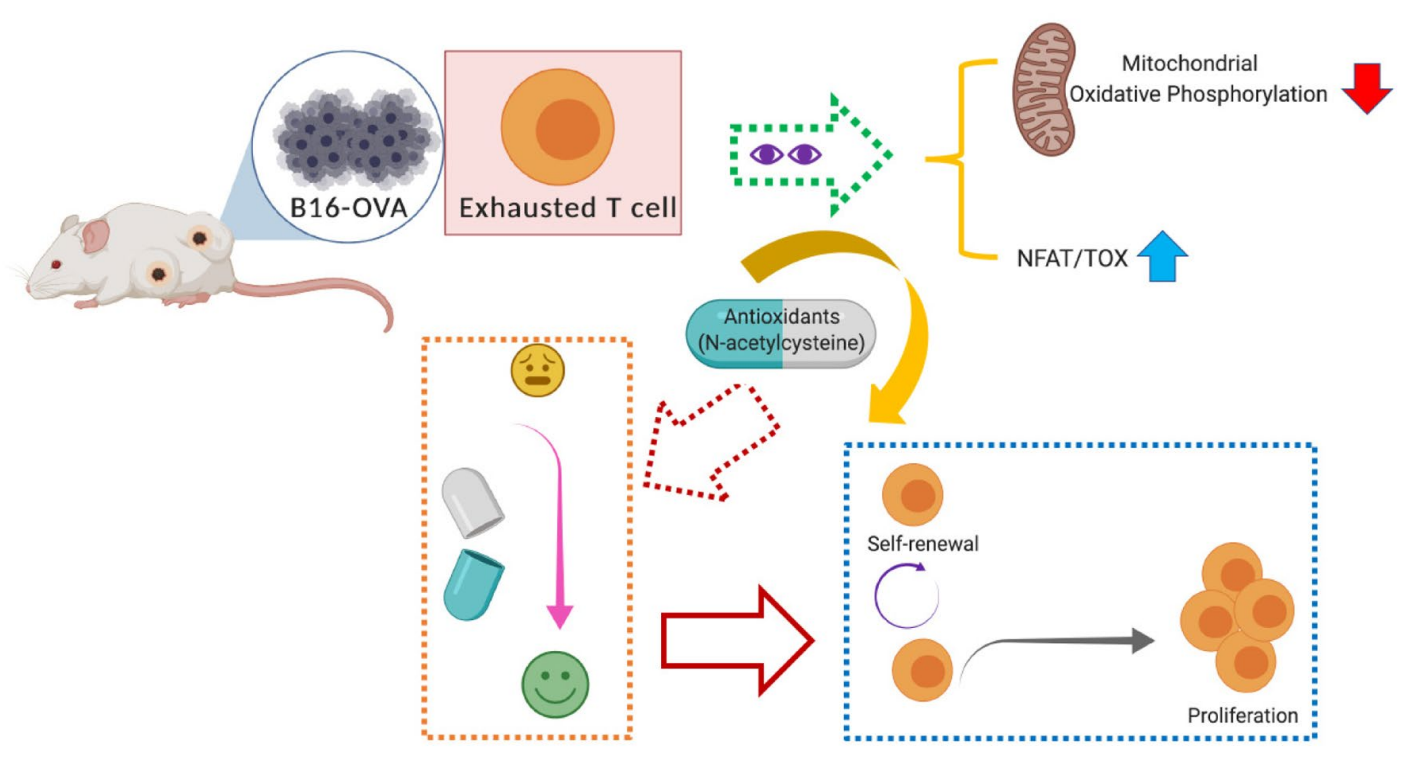

Figure 1. $\mathrm{N}$-acetylcysteine administration reinstates impaired mitochondrial OXPHOS in exhausted T cells, thus restoring the T-cell self-renewal and proliferation capacities in B16F10-OVA-challenged mice. 
Previous studies have emphasized the need to further characterize key factors controlling T-cell exhaustion to guide the development of future therapy to enhance anti-tumor immunity. Well-established examples include CTLA-4, PD-1, B7-H4, and CD28 [4]. For example, CD28 signaling can enhance glycolytic flux to satisfy the metabolic needs for T-cell activation [5], whereas PD-1 and CTLA-4 signaling impede T-cell activation by impairing glucose uptake [6]. Overcoming the restriction of T-cell exhaustion would be another critical means of enhancing anti-tumor therapy. In the new study, Vardhana et al. [3] additionally characterized how metabolic alterations influence exhausted $\mathrm{T}$ cells, and demonstrated that antioxidants such as Nacetylcysteine could reverse exhausted T-cell capacity and preserve anti-tumor $\mathrm{T}$-cell responsiveness in vivo. Unrestrained oxidative stress can result in protein and/or DNA destruction in cancer cells, and antioxidants (e.g., vitamin $\mathrm{C}$ and selenium) have been extensively utilized to counter these effects in cancer. However, vitamin $\mathrm{C}$ reportedly enhances the expansion of regulatory $\mathrm{T}$ cells (Tregs) [7], which decreases antitumor immunity. It is possible that $\mathrm{N}$-acetylcysteine could exert side-effects on other immune cell subsets, such as Tregs and dendritic cells (DCs). Thus, it is warranted to further dissect the global alterations of immunity during $\mathrm{N}$-acetylcysteine treatment. Ideally, further research will yield an optimal synergy strategy for boosting anti-tumor immunity against melanoma.

T-cell exhaustion is also reported in patients with hepatitis $\mathrm{B}$ virus (HBV) and hepatitis $\mathrm{C}$ virus (HCV), and it is unknown whether this occurrence is related to a metabolic mechanism. It would be interesting to analyze exhausted $\mathrm{HBV}$-specific $\mathrm{CD}^{+} \mathrm{T}$ cells by liquid chromatography-mass spectrometry (LC-MS) and single-cell RNA sequencing. Non-coding RNAs, such as microRNA-155, reportedly regulate exhausted $\mathrm{CD} 8^{+}$ $\mathrm{T}$ cells in models of chronic lymphocytic choriomeningitis virus (LCMV) infection [8]. It remains unclear how NFAT is modulated in exhausted T cells, and whether microRNA is involved in this process. Several potential microRNAs are predicted to target NFAT1, including microRNA-137 and microRNA-30$5 \mathrm{p}$. Future studies are needed to examine how Nacetylcysteine alters microRNA expressions, and how NFAT and the transcription factor TOX are regulated in exhausted T cells.

The new work by Vardhana et al. [3] connects mitochondrial dysfunction to exhausted $\mathrm{T}$ cells using in vitro chronic antigen stimulation and in vivo mouse models. Their findings indicate a potential new strategy for enhancing anti-tumor immunity against melanoma. Since PD-1/PD-L1 targeting has been utilized in triplenegative breast cancer (TNBC), the murine TNBC cell line 4T1 might be useful for testing the link between mitochondrial oxidative phosphorylation and selfrenewal of exhausted $\mathrm{T}$ cells upon persistent antigen challenge in future research.

\section{REFERENCES}

1. Patel CH, et al. Nat Rev Drug Discov. 2019; 18:669-88. https://doi.org/10.1038/s41573-019-0032-5 PMID:31363227

2. Buck MD, et al. J Exp Med. 2015; 212:1345-60. https://doi.org/10.1084/jem.20151159 PMID:26261266

3. Vardhana SA, et al. Nat Immunol. 2020; 21:1022-33. https://doi.org/10.1038/s41590-020-0725-2 PMID:32661364

4. Waldman AD, et al. Nat Rev Immunol. 2020. https://doi.org/10.1038/s41577-020-0306-5 PMID:32433532

5. Frauwirth KA, et al. Immunity. 2002; 16:769-77. https://doi.org/10.1016/S1074-7613(02)00323-0 PMID: $\underline{12121659}$

6. Intlekofer AM, Thompson CB. J Leukoc Biol. 2013; 94:25-39. $\quad$ https://doi.org/10.1189/jlb.1212621 PMID:23625198

7. Oyarce K, et al. Front Immunol. 2018; 9:112. https://doi.org/10.3389/fimmu.2018.00112 PMID:29479348

8. Stelekati E, et al. Cell Rep. 2018; 23:2142-56. https://doi.org/10.1016/i.celrep.2018.04.038 PMID:29768211

Shuai Jiang: Department of Microbiology and Immunology, Louisiana State University Health Sciences Center, Shreveport, LA 71130, USA

Correspondence: Shuai Jiang

Email: sjiang@Isuhsc.edu

Keywords: mitochondrial oxidative phosphorylation, $T$ cell exhaustion, NFAT, N-acetylcysteine

Funding: This study is supported by Louisiana State University (LSU) Feist-Weiller Cancer Center (FWCC) Legacy Funds (149741373A), Intramural Research Council Support (110251225A), and the Micro Seed Funds

Copyright: Jiang. This is an open-access article distributed under the terms of the Creative Commons Attribution License (CC BY 3.0), which permits unrestricted use, distribution, and reproduction in any medium, provided the original author and source are credited

Received: August 5, 2020

Published: September 7, 2020 